\title{
Analytical method for designing dispersion-managed fiber systems
}

\author{
K. Nakkeeran, A. B. Moubissi, and P. Tchofo Dinda \\ Laboratoire de Physique de l'Université de Bourgogne, Unité Mixte de Recherche du Centre National de \\ la Recherche Scientifique 5027, Avenue A. Savary, B.P. 47 870, 21078 Dijon Cédex, France \\ S. Wabnitz* \\ Alcatel Research \& Innovation, Route de Nozay, 91460 Marcoussis, France
}

Received May 30, 2001

\begin{abstract}
Using the equations of motion of pulse width and chirp, we present an analytical method for designing dispersion-managed (DM) fiber systems without optical losses. We show that the initial Gaussian pulse considered for the analytical design of periodically amplified DM fiber systems with losses will propagate as a proximity fixed point. Then averaging the DM soliton fields obtained from the slow dynamics of the proximity fixed point will yield the exact fixed point. () 2001 Optical Society of America

OCIS codes: $060.5530,190.5530$.
\end{abstract}

Recent studies have demonstrated that the use of dispersion-managed (DM) solitons for data transmission will substantially increase the capacity of fiber-optic links. ${ }^{1}$ Designing the dispersion map for a given transmission line characterized by specific pulse width and energy is probably a major step in setting up a DM soliton-based communication system. To our knowledge, all the techniques for designing the dispersion map of a DM system that have appeared so far in the literature rely on numerical procedures. In particular, the parameters of the stationary pulses (fixed point) for a given DM line are commonly obtained either by repeated solution of the nonlinear Schrödinger equation (NLSE) that describes the line or by solution of variational equations that correspond to the NLSE. Those numerical techniques for designing the dispersion map exhibit two major drawbacks. First, they are generally much too time consuming. In fact, the number of calculations required for obtaining a stationary pulse varies substantially, depending on the particular DM line under consideration. The general numerical procedure for finding a fixed point is to start with some arbitrary solution and achieve stationary propagation by periodically averaging out the pulse fields. But there is no guarantee of obtaining a stationary solution that corresponds to the desired pulse width and energy. Here lies the second drawback of the numerical procedure for designing a dispersion map of a DM fiber system. In this Letter we present a fully analytical procedure for designing a DM fiber system for any desired pulse and fiber parameters.

The pulse dynamics in DM fibers is governed by the NLSE:

$$
\psi_{z}+\frac{i \beta(z)}{2} \psi_{t t}-i \gamma|\psi|^{2} \psi=0
$$

where $\psi$ is the slowly varying envelope of the axial electrical field and $\beta(z)$ and $\gamma$ represent the groupvelocity dispersion and self-phase modulation parameters, respectively. First we consider the design of a
DM fiber system without losses. In our analytical design the fiber dispersion values are denoted $\beta_{+}$(normal) and $\beta_{-}$(anomalous). The input pulse width at the midpoint of the anomalous fiber $\left(x_{3-}\right)$, the energy $\left(E_{0}\right)$, and the maximum pulse width $\left(x_{3 m}\right)$ are the initial data required for deriving the lengths of anomalous $\left(L_{-}\right)$and normal $\left(L_{+}\right)$fibers for periodic evolution of the DM solitons. It is a well-known fact that the Gaussian ansatz provides an approximate but highly accurate representation of a DM soliton profile in a large parameter range including most practical situations. If we assume that the solution of the NLSE [Eq. (1)] is in the form of a Gaussian ansatz:

$$
\psi_{g}=x_{1} \exp \left[-\xi^{2} / x_{3}^{2}+i x_{4} \xi^{2} / 2+i x_{5} \xi+i x_{6}\right],
$$

where $\xi=t-x_{2}$ and $x_{1}, x_{2}, \sqrt{2 \ln 2} x_{3}, x_{4} /(2 \pi)$, $x_{5} /(2 \pi)$, and $x_{6}$ represent the pulse amplitude, temporal position, width (FWHM), chirp, frequency, and phase, respectively, then, by using the variational method, we derive the equations for the pulse width and chirp as

$$
\begin{aligned}
& \dot{x}_{3}=-\beta(z) x_{3} x_{4}, \\
& \dot{x}_{4}=\beta(z)\left(x_{4}{ }^{2}-4 x_{3}{ }^{-4}\right)-\sqrt{2} \gamma E_{0} x_{3}{ }^{-3} .
\end{aligned}
$$

Here $E_{0}=x_{1}^{2} x_{3}$ is a constant that represents the energy of the Gaussian pulse. Taking the derivative of Eq. (3a) with respect to $z$ and using Eq. (3b), we derive the equation for pulse duration as

$$
\ddot{x}_{3}=4 \beta(z)^{2} x_{3}{ }^{-3}+\sqrt{2} \beta(z) \gamma E_{0} x_{3}{ }^{-2} .
$$

Integrating Eq. (4) with respect to $x_{3}$, we get

$$
\dot{x}_{3}^{2} / 2=-2 \beta(z)^{2} x_{3}{ }^{-2}-\sqrt{2} \beta(z) \gamma E_{0} x_{3}^{-1}+c .
$$

The constant of integration, $c$, can be different for normal $\left(c_{+}\right)$and anomalous $\left(c_{-}\right)$fiber sections of the 
dispersion map. $c_{ \pm}$can be evaluated directly at the midpoint of each section of the fiber where the pulse duration reaches its minimum as

$$
c_{ \pm}=2{\beta_{ \pm}}^{2} x_{3 \pm}{ }^{-2}+\sqrt{2} \beta_{ \pm} \gamma E_{0} x_{3 \pm}{ }^{-1} .
$$

Grigoryan et al. considered a similar kind of constant at the fiber junctions and used numerical techniques to describe the dependence of DM soliton energy on the average dispersion. ${ }^{2}$ Chen and Haus derived the same equations for the constants $c_{ \pm} .{ }^{3}$ Here we derive the equation that relates these constants $\left(c_{ \pm}\right)$ to the maximum pulse width. For that we derive the expression for the maximum pulse width $\left(x_{3 m}\right)$ from the continuity of chirp when the DM soliton propagates from one fiber section to another fiber section. Then, at the fiber junctions from Eq. (3a), we have

$$
\left.\frac{\dot{x}_{3-}}{\beta_{-} x_{3-}}\right|_{x_{3 m}}=\left.\frac{\dot{x}_{3+}}{\beta_{+} x_{3+}}\right|_{x_{3 m}} \text {. }
$$

From Eqs. (5) and (7) we obtain the following expression for the maximum pulse width:

$$
x_{3 m}=\sqrt{2} \gamma E_{0} \beta_{+} \beta_{-}\left(\beta_{-}-\beta_{+}\right) /\left(c_{+} \beta_{-}{ }^{2}-c_{-} \beta_{+}{ }^{2}\right) .
$$

To derive the expression for the length of the fiber sections, we must integrate Eq. (5) with respect to $z$. Then we find the length of normal $\left(L_{+}\right)$and anomalous $\left(L_{-}\right)$fiber sections:

$$
\begin{aligned}
\frac{L_{ \pm}}{2}= & f\left(\beta_{ \pm}, c_{ \pm}, x_{3 m}\right)-\frac{\gamma \beta_{ \pm} E_{0}}{2 c_{ \pm} \sqrt{c_{ \pm}}} \ln \left(4 c_{ \pm} x_{3 \pm}\right. \\
& \left.-2 \sqrt{2} \gamma \beta_{ \pm} E_{0}\right)
\end{aligned}
$$

where $f\left(\beta_{ \pm}, c_{ \pm}, x_{3}\right)=\left[R_{ \pm}\left(x_{3}\right)\right]^{1 / 2} / 2 c_{ \pm}+\gamma \beta_{ \pm} E_{0} / 2 c_{ \pm} \times$ $\sqrt{c_{ \pm}} \ln \left\{2\left[2 c_{ \pm} R_{ \pm}\left(x_{3}\right)\right]^{1 / 2}+4 c_{ \pm} x_{3}-2 \sqrt{2} \gamma \beta_{ \pm} E_{0}\right\}$, with $R_{ \pm}\left(x_{3}\right)=2 c_{ \pm} x_{3}{ }^{2}-2 \sqrt{2} \beta_{ \pm} \gamma E_{0} x_{3}-4{\beta_{ \pm}}^{2}$. Thus, using the data $x_{3-}, E_{0}, \beta_{ \pm}$, and $\gamma$, we can calculate the constant $c_{-}$from Eq. (6). Then, using the value of $x_{3 m}$ and $c_{-}$, we can calculate the other constant, $c_{+}$, from Eq. (8). After that the value of $x_{3+}$ can be directly obtained from Eq. (6). Then, using Eq. (9), we can straightforwardly evaluate the lengths of the normal and anomalous fiber sections. We verified this analytical design for lossless DM fiber lines and found that it works extremely well, as a Gaussian ansatz well describes the single DM soliton dynamics. ${ }^{4}$ The main virtue of this analytical approach is that one can design the DM system to correspond to any desired pulse with energy $E_{0}$ and width $x_{3-}$. A second advantage is that the amount of calculations required for the analytical design represents an extremely small fraction of the amount of calculations one needs to obtain the fixed point directly from a pseudodynamic relaxation by using variational equations (3).

To illustrate the analytical design procedure, we take the following input data: initial pulse width, $x_{3-}=10 \mathrm{ps}$; maximum pulse width, $x_{3 m}=16.63 \mathrm{ps}$; energy, $E_{0}=0.2 \mathrm{pJ}$; dispersion, $\beta_{ \pm}= \pm 6.8 \mathrm{ps}^{2} / \mathrm{km}$; and Kerr coefficient, $\gamma=0.0014 \mathrm{~mW}^{-1}$. From Eqs. (6),
(8), and (9) we directly obtain $L_{-} / 2=10.6633 \mathrm{~km}$ and $L_{+} / 2=9.3367 \mathrm{~km}$. Figure 1 shows the closed loop formed from the plot of the pulse width relative to chirp obtained from propagation of the initially assumed Gaussian pulse in the analytically designed dispersion map. A useful aspect of this analytical design is that it can be exploited to yield the proximity fixed point for a DM system in the presence of losses and gain, where a proximity fixed point is defined as the Gaussian pulse that will propagate without any radiation and during propagation will periodically return to the initial pulse width and chirp after a certain number of dispersion maps. The ideal fixed point, however, will have the same width and a chirp after each dispersion map.

For the analytical design with optical losses it is worth recalling that, because of the fiber losses, the fixed point of a symmetric map has some initial chirp. This initial chirp, after a small distance of propagation, say, $\delta$, will become zero. One can cut this length of fiber $\delta$ from the first section of the symmetric map and merge it at the end of the map. So the resultant map will be a modified symmetric map, whose fixed point will initially be unchirped. ${ }^{5}$ In what follows, we show that our analytical design results in a similar kind of modified symmetric map for a system with losses and gain. Let us first consider the analytical design of a DM fiber line in a particular case for which there is only one map in each amplification period. As a first step in the analytical design of this system with losses, by using the above analytical procedure we derive the fiber lengths $\left(L_{ \pm}\right)$of a symmetric map without losses. We split that symmetric map into two asymmetric maps with equal total lengths $L=\left(L_{+}+L_{-}\right) / 2$. We assume that energy $E_{0}$ is almost constant in the first asymmetric map. Because of the optical losses, the energy available at the input of the second asymmetric map will be $E_{0} \exp (-\alpha L)$, where $\alpha$ is a loss parameter of both types of fiber. So we derive the new fiber lengths $L_{ \pm}{ }^{\prime} / 2$ for the second asymmetric map with the same

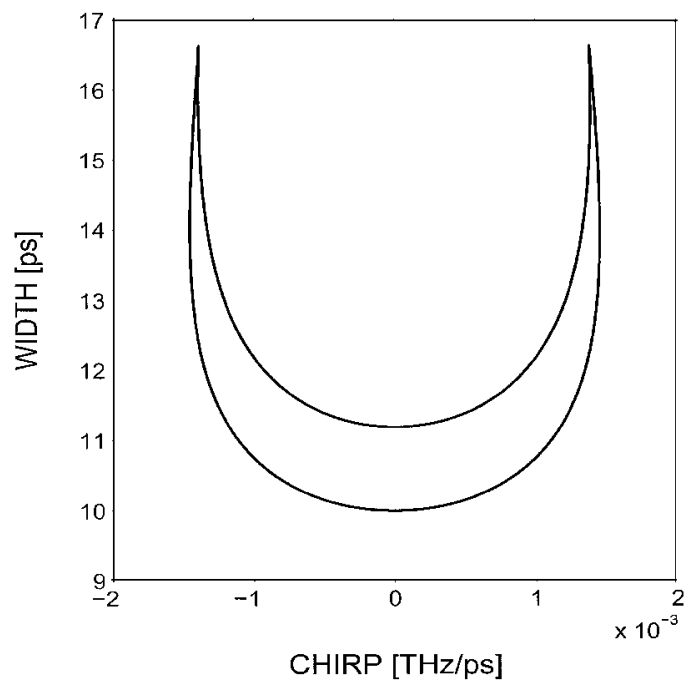

Fig. 1. Pulse width versus chirp obtained from the propagation of an initially assumed Gaussian pulse in the analytically design map without losses. 


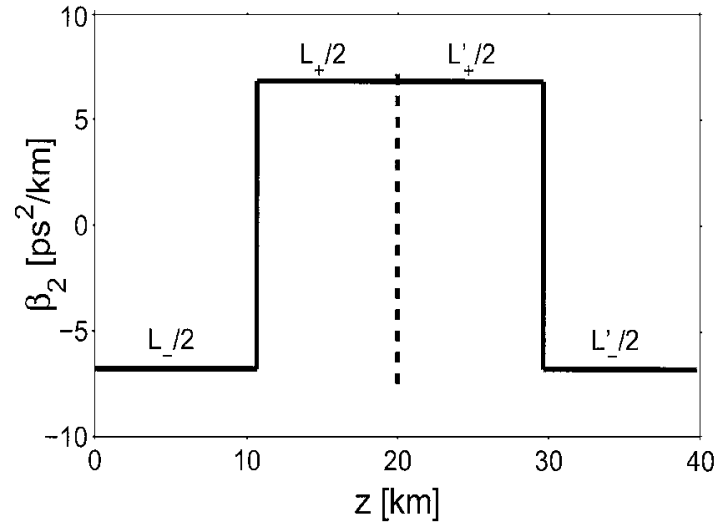

Fig. 2. Schematic representation of the analytically designed dispersion map with losses and gain.

Propagation of proximity fixed point

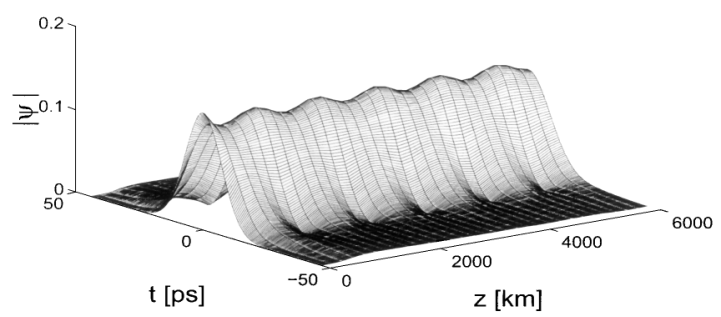

Propagation of fixed point

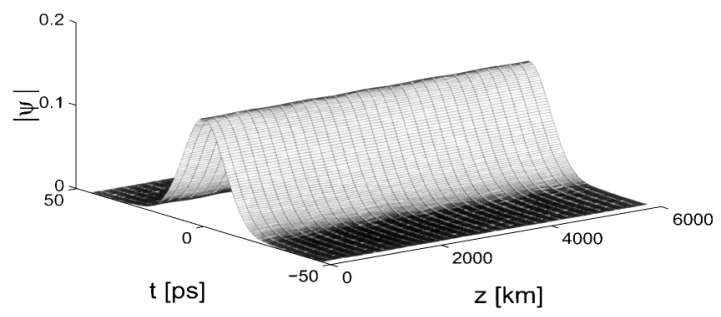

Fig. 3. Evolution of the proximity DM soliton field and the averaged DM soliton field plotted after each amplification period for propagation in the analytically designed map with losses and gain. $\beta_{ \pm}= \pm 6.8 \mathrm{ps}^{2} / \mathrm{km}$, $\gamma=0.0014 \mathrm{~mW}^{-1}$, and $\alpha=0.2 \mathrm{~dB} / \mathrm{km}$.

initial data that we used to design the initial symmetric map, except for the energy. Then simply merging the two asymmetrical maps gives the analytical design procedure for the modified symmetric map with periodic losses and amplification. Hence the final design may have $L_{-} / 2$ length of anomalous fiber followed by $L_{+} / 2+L_{+}{ }^{\prime} / 2$ length of normal fiber and finally $L_{-}^{\prime} / 2$ length of anomalous fiber. Figure 2 shows a schematic diagram of the analytically designed dispersion map. For analytically designing DM fiber lines with more than one periodic dispersion map in one amplification, we consider that the energy within every dispersion map is a constant. Energy is considered to be stepwise exponentially decreasing from one map to the other. So we can calculate the lengths of anomalous and normal fiber sections of individual dispersion maps by using the same initial data, except for the energy. We also find that the Gaussian pulse with initial data (width $x_{3-}$ and energy $E_{0}$ ) propagates as a proximity fixed point in the analytically designed DM fiber lines with losses and gain. When we average ${ }^{6}$ the DM soliton fields obtained from the slow dynamics of the proximity Gaussian fixed point, we immediately obtain the fixed point for the periodically amplified DM fiber system with losses. A fundamental property of this analytical design is that the proximity fixed point gets closer to the exact fixed point as the number of maps within an amplification period increases.

To demonstrate the procedure for analytical design of a DM fiber system with losses and gain, we consider a system with one dispersion map in one amplification period. For that, we consider the same input data as in the above design of a lossless DM fiber system and a loss parameter (for both normal and anomalous fibers) $\alpha=0.2 \mathrm{~dB} / \mathrm{km}$. From our analytical procedure we have the fiber lengths of two asymmetric maps as $L_{-} / 2=10.6633 \mathrm{~km}, L_{+} / 2=$ $9.3367 \mathrm{~km}, L_{-}{ }^{\prime} / 2=10.098 \mathrm{~km}$, and $L_{+}{ }^{\prime} / 2=9.632 \mathrm{~km}$. Hence the amplification period length becomes $\left(L_{-} /\right.$ $\left.2+L_{+} / 2+L_{+}{ }^{\prime} / 2+L_{-}^{\prime} / 2\right) 39.73 \mathrm{~km}$. Figure 3 shows the evolution of the proximity Gaussian fixed point and the fixed point (obtained from averaging ${ }^{6}$ the DM soliton fields of the slow dynamics of the proximity fixed point) for $6000 \mathrm{~km}$ in the analytically designed modified symmetric map with losses and gain.

In conclusion, we have presented a fully analytical method for designing DM fiber systems. By this analytical procedure one can easily and efficiently design DM fiber transmission lines with periodic losses and gain for any desired pulse parameters.

This study has been carried out under contract URP/4.00 between the University of Burgundy and the Alcatel Research Corporation (URP/C/01/0146). We gratefully acknowledge partial support of this research from the Ministère de l'Education Nationale de la Recherche et de la Technologies (contract ACI Jeunes 2015). K. Nakkeeran thanks the Centre National de la Recherche Scientifique for offering a research associate fellowship. K. Nakkeeran's e-mail address is naks@jupiter.u-bourgogne.fr.

*Present address, Xtera Communications, 500 West Bethany Drive, Allen, Texas 75013.

\section{References}

1. V. E. Zakharov and S. Wabnitz, Optical Solitons: Theoretical Challenges and Industrial Perspectives (Springer-Verlag, Berlin, 1998).

2. V. S. Grigoryan, T. Yu, E. A. Golovchenko, C. R. Menyuk, and A. N. Pilipetskii, Opt. Lett. 22, 1609 (1997).

3. Y. Chen and H. A. Haus, J. Opt. Soc. Am. B 16, 24 (1999).

4. P. Tchofo Dinda, A. B. Moubissi, and K. Nakkeeran, J. Phys. A 34, L103 (2001).

5. T. S. Yang, W. L. Kath, and S. K. Turitsyn, Opt. Lett. 23, 597 (1998).

6. J. H. B. Nijhof, N. J. Doran, W. Forysiak, and F. M. Knox, Electron. Lett. 33, 1726 (1997). 